\title{
Tratamiento con pentoxifilina oral para las úlceras venosas de los miembros inferiores
}

Systemic treatment of venous leg ulcers with high doses of pentoxifyline in a randomized placebo-controlled trial. Falanga V, Fujitani R, Díaz C, et. al. Wound rep reg 1999;7:208-213.

\begin{abstract}
Objetivos
Investigar la eficacia y la dosificación óptima (400 ú $800 \mathrm{mg}$ tres veces por día) de la pentoxifilina (PTX) oral para promover la cicatrización de las úlceras venosas de los miembros inferiores (UVMI).

\section{Métodos}

Estudio multicéntrico doble ciego randomizado con placebo que incluyó tres ramas a una dosificación de tres veces por día: 1) placebo, 2) $400 \mathrm{mg}$ de pentoxifilina, 3) $800 \mathrm{mg}$ de pentoxifilina. Todos los pacientes recibieron además el tratamiento usual de compresión graduada con un vendaje impregnado con zinc.
\end{abstract}

\section{Lugar}

14 centros de Estados Unidos.

\section{Pacientes}

131 pacientes divididos en tres grupos.

Criterios de inclusión: una o más úlceras venosas de por lo menos $1 \mathrm{~cm}$ de diámetro con más de dos y menos de 24 meses de duración, manifestaciones clínicas de insuficiencia venosa (localización medial, hiperpigmentación, lipodermatoesclerosis, várices) confirmada por ecografía doppler de las venas superficiales y profundas de ambos miembros inferiores, 18 a 90 años de edad y ausencia de insuficiencia arterial significativa de los miembros inferiores documentada por un índice tobillo-brazo de TA mayor a 0.5, posibilidad de deambular y un peso corporal que se encontrara entre el 90 y el $150 \%$ del ideal. Criterios de exclusión: embarazo, alergia a las xantinas, simpatectomía lumbar en los últimos tres meses, hemoglobina glicosilada mayor de $10 \%$, úlceras por diabetes o isquemia o con exposición tendinosa o que requirieran antibióticoterapia sistémica, así como antecedentes de mala adherencia a los tratamientos.

\section{Resultados}

Se realizó el análisis por intención de tratar. Se perdieron en el seguimiento 10 pacientes del grupo placebo, 11 del de $400 \mathrm{mg}$ de PTX y 11 del de $800 \mathrm{mg}$. Las diferencias en la efectividad de los tratamientos comenzaron a notarse en la octava semana. Los pacientes que habían recibido las tres dosis de $800 \mathrm{mg}$ evidenciaron una mediana de tiempo* a la cicatrización completa de 71 días mientras que en los del grupo placebo este tiempo fue de 100 días $(p=0.043)$. Si bien están inscriptos en la tendencia esperable, no hubo diferencias estadísticamente significativas entre el grupo placebo y el de $400 \mathrm{mg}$ de PTX, cuyos pacientes tuvieron un tiempo medio de cicatrización de 83 días.

No hubo diferencias significativas en la incidencia de efectos adversos.

\section{COMENTARIO}

Para el manejo de las UVMI se han ensayado muchas estrategias terapéuticas. Las medidas generales para mejorar el retorno venoso y disminuir el edema incluyen la elevación intermitente del/los miembro/s inferiores ${ }^{1}$ y la compresión graduada de la extremidad ${ }^{2}$ a través de una media o venda elástica (contraindicado si el paciente tiene enfermedad arterial significativa). Ambas han demostrado acelerar la velocidad de curación y disminuir la recurrencia de las UVMI. Los pacientes con enfermedad arterial o quienes no toleran las medias elásticas podrían mejorar con extracto de Castaña de la India, que ha demostrado reducir el edema y los síntomas de insuficiencia venosa ${ }^{3}$, o bien con algún derivado de los rutósidos (si bien hay reportes de que su eficacia es superior al uso de placebo, la evidencia es algo más controvertida $)^{1}$. Si el edema es clínicamente manifiesto un curso de unos pocos días de diuréticos tiazídicos puede ser de utilidad.

Los pacientes sin contraindicaciones para recibirla, pueden beneficiarse con aspirina en dosis antiagregantes ${ }^{4}$, mientras que cuando hay signos clínicos de infección está indicada la antibióticoterapia (muchos expertos desalientan su uso rutinario, aún en forma tópica, para evitar la colonización con gérmenes resistentes). En cuanto a la higiene de la úlcera se desaconseja el uso de iodopoviona por sus efectos citotóxicos, recomendándose el de agua oxigenada 1 .

Respecto de los hallazgos de este trabajo llama la atención las pérdidas en el seguimiento, que llegaron casi al $25 \%$ de los pacientes, lo que podría invalidar los resultados obtenidos (ej. si los pacientes que se perdieron del grupo de $800 \mathrm{mg}$ de PTX hubieran sido los que peor respondieron al tratamiento podríamos estar sobrevalorando sus efectos beneficiosos). Como informan los autores de este trabajo, la mediana de tiempo a la curación fue de 71 días en los pacientes tratados con $800 \mathrm{mg}$ de PTX y de 100 días en los que recibieron placebo. Vale la pena aclarar que a las 24 semanas de tratamiento las diferencias son algo menores ya que en ese tiempo han logrado la curación total el $73 \%$ de los pacientes tratados con PTX (tanto a 400 como a $800 \mathrm{mg}$ por dosis) y el $63 \%$ de los tratados con placebo, lo que evidenciaría que es necesario tratar 10 pacientes durante 24 semanas para lograr una curación adicional en dicho lapso.

Siguiendo esta misma línea de investigación, una muy prolija y reciente revisión sistemática ${ }^{5}$ de la Colaboración Cochrane (que incluyó a este trabajo) informó resultados concordantes luego de realizar un metanálisis de la evidencia encontrada. Sin embargo, esto no ocurrió con la dosificación ya que que, exceptuando el estudio que aquí comentamos, el resto de los incluidos en dicha revisión habían utilizado sólo $400 \mathrm{mg}$ de PTX por dosis. Esta revisión concluye que el uso de $400 \mathrm{mg}$ de PTX cada ocho horas durante 24 semanas sumado al tratamiento compresivo estándar aumenta la probabilidad de curación (RR=1.3; IC95\%: 1.1-1.54) necesitándose tratar 7 pacientes (IC95\%: 4 a 17) en dicho lapso para lograr una curación adicional. Teniendo en cuenta el costo del tratamiento (aproximadamente $\$ 110$ por mes a una dosis de $400 \mathrm{mg}$ cada 8 horas y el doble a la de $800 \mathrm{mg}$ en Argentina) vale la pena considerar y discutir con los pacientes los potenciales beneficios del mismo para poder decidir o no su uso y para elegir la mejor dosificación, que por el momento pareciera seguir siendo $400 \mathrm{mg}$ cada ocho horas.

\section{Dr. Sergio Terrasa}

*Ver glosario

Unidad de Medicina Familiar y Preventiva del Hospital Italiano de Buenos Aires.

\section{Referencias}

1.Alguire P, Mathes B. Treatment of chronic venous insufficiency. En Burton Rose, editor in Chief, Up to date. Vol 8. No 2. www.uptodate.com

2.NHS Centre of Reviews and Dissemination, University of York. Effective Health Care, Compression Therapy for venous leg ulcers. August 1997. Vol 3. number 4. ISSN: $0965-0288$. 3.Diehm c, Trampisch H, Lange S, et. al. Comparisson of leg compression stokings and oral horse chestnut seed extract therapy in patients with chronic venous insufficiency. Lancet 1996, 347.292

4.Layton A, Ibbotson S, Davis J, et al. Randomised trial of oral aspirin for chronic venous leg ulcers. Lancet 1994, 344-164.

5.Jull A, Waters J. Oral pentoxifyline for treatment of venous leg ulcers. The Cochrane Database of Systematic Reviews (ISBN 190186805 2) 3: 00587, 2000). Disponible gratuitamente en internet en www.obgyn.net. 